\section{La importancia de la estadística para el éxito de resultados en investigación}

\section{The importance of statistics for successful research results}

Alex Darío Jacho Guashca

Estudiante, Escuela Superior Politécnica de Chimborazo, Riobamba, Ecuador, alex.jacho@espoch.edu.ec, https://orcid.org/ooooooo2-8759-5486

\section{Ivan Holger Loaiza Campoverde}

Estudiante, Escuela Superior Politécnica de Chimborazo, Riobamba, Ecuador, ivan.loaiza@espoch.edu.ec, https://orcid.org/oooo-0003-4263-6122

\section{José Luis López Salazar}

Master en investigación en Administración y Economía de la Empresa, Escuela Superior Politécnica de Chimborazo, Riobamba, Ecuador, lopezsalazarjl@gmail.com, https://orcid.org/ooooooo3-1012-4472

\section{Resumen}

El presente artículo abarca el análisis sobre el papel que juega la estadística en la investigación, partiendo de una exhaustiva revisión bibliográfica, donde se identifica conceptos básicos para una mejor comprensión y entendimiento del contenido. Se utilizó una metodología cualitativa que nos permitió determinar las principales características, funciones e importancia para que la estadística sea fundamental en la obtención de resultados precisos que nos

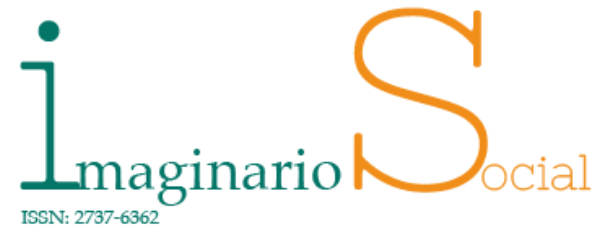

Imaginario Social

e-ISSN: $2737-6362$

enero - junio 2020 Vol. 3-1-2020

http://revista-

imaginariosocial.com/index.php/es/in dex

Recepción: 13 de agosto 2019

Aceptación: 14 de octubre 2019

32-41

Atribución/Reconocimiento-NoCo mercial- Compartirlgual 4.0 Licencia Pública Internacional - CC BY-NC-SA 4.0

https://creativecommons.org/licenses /by-nc- sa/4.0/legalcode.es 
permita tomar las mejores decisiones al término de una investigación. La información cuantitativa nos permite realizar una investigación apegada a la realidad ya que, a través de números, estadísticas, censos y otros datos podemos precisar resultados concretos y de esta manera nos permite prever acontecimientos que puedan suscitar en un futuro. El papel que juega la estadística en la investigación es oportuno y de gran relevancia para que se logre el alcance de los objetivos dentro de un estudio propuesto.

Palabras clave: estadística, investigación, información, análisis cuantitativo, análisis cualitativo.

\begin{abstract}
This article covers the analysis of the role that statistics play in research, based on an exhaustive bibliographic review, where basic concepts are identified for a better understanding and comprehension of the content. We used a qualitative methodology that allowed us to determine the main characteristics, functions and importance for statistics to be fundamental in obtaining precise results that allow us to make the best decisions at the end of a research. Quantitative information allows us to carry out research that is attached to reality since, through numbers, statistics, censuses and other data, we can specify concrete results and, in this way, we can anticipate events that may arise in the future. The role that statistics play in research is timely and of great relevance to the achievement of the objectives within a proposed study.
\end{abstract}

Key word: statistics, research, information, quantitative analysis, qualitative analysis.

\title{
Introducción
}

La estadística es una ciencia que reúne información cuantitativa de ciertos hechos, individuos o grupos, y se deduce mediante un análisis, resultados precisos o previsiones para el futuro, permitiendo tomar las mejores decisiones de una situación, acontecimiento o investigación. El origen de la Estadística se remonta a los comienzos de la historia y esto se sabe tanto a través de las representaciones, tanto gráficas y simbólicas que se encontraron en pieles, rocas, palos de madera y paredes de cuevas para contar el número de personas, animales o ciertas cosas 
que eran necesarias para efectuar los respectivos cálculos (Ponteiro, 2013). El mayor desenvolvimiento de la estadística surgió en el área de las ciencias naturales cuando era necesario interpretar datos a través del uso de herramientas analíticas; un ejemplo claro se da en la agricultura donde se requiere el uso de dichas herramientas estadísticas para que la productividad de cosechas mantenga un gran rendimiento (Foroughbakhch \& M, 2005).

La estadística juega un papel muy importante para obtener resultados, en el siglo XXI la globalización ha crecido aceleradamente, y las investigaciones son realmente necesarias para conseguir resultados y prever situaciones a futuro. Entonces, se podría decir que ċla estadística es importante para realizar una investigación? ¿qué resultados podemos obtener si aplicamos la estadística en el ámbito demográfico? ¿Es realmente necesario su utilización para determinar la eficacia de una investigación?

La presente investigación tiene la finalidad de aclarar estas interrogantes y de analizar el papel que juega la estadística en la investigación enfatizando las características que nos aporta para que podamos obtener resultados confiables y de esa manera tomar decisiones eficientes. Además, se presentarán conceptos que nos ayuden a dar un mejor entendimiento del contenido y las pautas necesarias para el seguimiento de la investigación, que brinde resultados eficientes y confiables en la actualidad.

\section{Materiales y Métodos}

Para la presente investigación se utilizó la metodología cualitativa, Taylor y Bogdan señalan que "es aquella que produce datos descriptivos: las propias palabras de las personas, habladas o escritas, y la conducta observable” (Taylor \& Bogdan, 1986). Esta investigación busca demostrar cómo la estadística es de gran relevancia para alcanzar resultados eficaces y a su vez mostrar datos reales que permitan sustentar y dar confiabilidad a las conclusiones obtenidas. La metodología cualitativa también "es una categoría de diseños de investigación que extraen descripciones a partir de observaciones que adoptan la forma de entrevistas, narraciones, notas de campo, grabaciones, transcripciones de audio y video casetes, registros escritos de todos tipo, fotografías o películas y artefactos" (Lecompte, 1995). Es así, que la información presentada en esta investigación fue extraída de artículos de internet, libros y revistas de fuentes confiables, lo cual nos permitió obtener conocimientos valiosos para sustentar nuestras conclusiones. 
Finalmente, se busca aportar con comprensiones propias, partiendo de pautas que ya han sido elaboradas, Hernández Zapata señala que "Es el método en el cual los investigadores parten de hechos particulares o concretos para llegar a conclusiones generales” (Zapata, 2017). De esta manera se brindará información nueva y sobre todo se resolverá la interrogante planteada de nuestra investigación a través de resultados claros y entendibles.

\section{Resultados}

\section{Estadística}

"Al hacer referencia a la palabra estadística, pocas son las personas las que tienen una definición clara acerca del término, se determina que la estadística es aquella que se la asocia con una serie de datos o columna de números; de acontecimientos que se dieron en el pasado o de hechos históricos o una lista de defunciones, nacimientos, causas de muerte en una región, en un periodo de tiempo determinado, listados de precios de un sin número de artículos, entre otras." (Parias, 2011). De la misma manera se puede determinar que los individuos al no definir una idea clara acerca de la estadística, son aquellas mismas que la interpretan de una manera inadecuada, provocando así la mala interpretación de la información.

"La estadística es una ciencia aplicada de las matemáticas y es una valiosa herramienta para la toma de decisiones. Permite el estudio de fenómenos mediante la descripción del mismo a través de inferencias mediante distribución probabilísticas" (Mendiburu, 2018). Al enfatizar que la estadística es aquella que comprende que, es una herramienta que tiene gran relevancia y es misma que se encarga de la recolección, agrupación, representación, análisis e interpretación de datos, permitiendo de esa manera facilitar la comprensión de los datos de una manera clara y concisa. La estadística no son solo aquellos resultados de encuestas, de la misma manera es una técnica científica que procura determinar conclusiones a partir de observaciones realizadas.

\section{Investigación}

"Es un procedimiento reflexivo, sistemático, controlado y crítico que tiene por finalidad descubrir hechos, fenómenos y leyes” (Delgado, 2013). La investigación es la búsqueda de información verídica para brindar posibles soluciones a los problemas que se hayan planteado, para eso se debe realizar procedimientos sistematizados que ayuden a dar un entendimiento claro y entendible a los resultados que se hayan conseguido. 
"La investigación tiene como principales objetivos la generación de conocimiento y la solución de problemas prácticos" (Manterola \& H., 2013). También se puede identificar a la investigación como aquella acción innata que cada persona posee frente a cualquier situación, fundamentándose en una serie de preguntas hasta poder determinar la solución de cuándo, dónde, cómo y por qué ocurrió cada hecho, de esa manera que le permite comprender cada situación en la que llegue a encontrarse.

La investigación también se puede considerar como aquella acción o efecto de realizar actividades intelectuales y experimentales, con el propósito de aumentar los conocimientos sobre un determinado tema o entorno en el que llegue a encontrarse la persona, con la finalidad de ampliar el conocimiento propio. Haciendo énfasis, de tal manera es un claro ejemplo de plantear problemas y de la misma manera la búsqueda de la respuesta mediante una búsqueda minuciosa de interés o una preocupación frente alguna circunstancia, investigando sobre hechos, datos, procesos, dificultades, inquietudes, entre otras. de un aspecto de la realidad, para de esa manera poder solucionarlo.

Como lo indica Merton, la investigación científica es aquella que tiene dos rasgos propios que la diferencian de las demás acciones que realiza el ser humano:

- Características internas, expresadas en la exigencia de congruencia lógica y de confirmación empírica, que son exigencias fundamentales del método científico y, consecuentemente, de la investigación que éste utiliza.

- Características institucionales, que se expresan en el comportamiento de la ciencia, constituido por el empleo de valores y normas que se consideran obligatorias para el hombre de ciencia, no porque hayan sido codificados, sino por consenso mora. (Merton, 1964).

\section{La estadística principal herramienta influyente en la investigación}

En la actualidad la estadística resulta ser un instrumento de trabajo útil en la investigación, la misma que ha tenido una gran acogida por parte de las personas, siendo una herramienta de investigación en los diferentes ámbitos en la medida en que ofrece técnicas y procedimientos que pueden ser aplicados en la etapa de análisis de datos, de la misma manera, permite una mejor comprensión de la investigación. Aceptando que esta es la aplicación que mayor énfasis tiene en el contexto en la investigación, de tal manera que existen diferentes ámbitos (Flores, 2003).

"La Estadística también se puede considerar que está presente desde la formulación del problema de investigación" (Gómez Benito, 1987). Desde esta perspectiva, el planteamiento de la problemática se determina en buena medida la recolección de datos que lleguen a ser necesarios, las diferentes técnicas eficientes para la recolección de la misma y los diferentes procedimientos estadísticos que se deban utilizar en el análisis respectivo. Por otra parte, 
menciona que entre las características que debe poseer cualquier problema de investigación se encuentra su re solubilidad, situación que en su determinado tiempo es garantizada si lo manipulamos con métodos adecuados de estadística, mismas que son capaces de abordar los enigmas de partida (Sánchez, 1989).

La Estadística llega a dividirse en:

Estadística descriptiva: es aquella que trata de la tabulación de datos, su presentación en forma gráfica o ilustrativa y el cálculo de medidas descriptivas.

Estadística inferencial: se llega a definir como aquellos métodos que permiten hacer estimación de una característica de la población o la determinación de disposiciones acerca de la población en sí, enfatizando de tal manera en los resultados obtenidos de una muestra. (Barreto-Villanueva, 2012)

Se puede determinar que en pleno siglo XXI la importancia que genera la estadística es de suma importancia debido a que esta permite la facilidad de la comprensión de la información y actúa en diferentes ámbitos en la sociedad, como en los siguientes ámbitos:

Ámbito Educación: Al existir estrechos vínculos entre la Estadística y la investigación pedagógica, lo que contribuye al entendimiento de las diferentes técnicas de investigación en la educación, el diseño de programas, las dificultades existentes para una correcta medición y control, el análisis respectivo y la correcta orientación.

Ámbito Económico: Se basa en la manipulación de diferentes datos numéricos. De tal manera que se logre una correcta interpretación y valoración con las respectivas herramientas estadísticas. Entre los más utilizados se puede mencionar; el índice de precios al consumo, el análisis de mercados, la evaluación de la demanda y las especias temporales.

Ámbito Demográfico: Siendo aquella encargada del estudio que se realiza a la población en general, mediante herramientas como es el censo, mismo que se lo realiza cada 10 años, siendo una información que permite la distribución por edades o sexo, localización geográfica, profesiones, religión, nacionalidades, tasas de nacimiento o defunción, movimientos sociales migratorios, entre otras. La estadística siendo una herramienta eficiente para el correcto perfilamiento y desarrollo de estas tareas.

Ámbito de Administración pública: la correcta interpretación de los datos generados por el censo de habitantes, su distribución, las diferentes fuentes de riquezas, los diferentes temas laborales y sectoriales existentes. El conocimiento en sí de todo esto sirve para una correcta planificación y toma de sesiones que son sumamente necesarios para cada zona, de tal manera que se pueda incentivar a que existe un bienestar social, generando así el desarrollo de una manera eficaz con el apoyo de las herramientas estadísticas que es de gran apoyo (BarretoVillanueva, 2012). 
En la presente investigación al identificar diferentes ámbitos de la sociedad en la que se apoya la estadística, generaremos un análisis a mayor profundidad en el ámbito demográfico.

Para poder generar un estudio basado en datos verídicos, debe acudir a la herramienta denominada censo, misma que es de gran relevancia para poder determinar los diferentes datos estadísticos, facilitando de tal manera la interpretación de información para una mejor toma de decisiones. Se puede recalcar también que los censos son recuentos exhaustivos de la población en general, que el estado obliga a que se realice de manera periódica, habitualmente esto se lo hace cada 10 años, para de esa manera poder determinar cuáles son las características de la sociedad y ámbitos demográficos de sus habitantes. Simultáneamente los censos de la población en general se efectúan en vivienda, de tal manera que permite identificar y relacionar las diferentes características de las viviendas con los habitantes que ocupan.

El censo es de suma importancia, misma que tiene diferentes finalidades benéficas. De la información recaudada gracias al censo se puede determinar conclusiones que sean de gran beneficio para una futura toma de decisiones, información como cuantos somos y de qué manera estamos distribuidos, el sexo, la edad, el lugar de nacimiento y residencia, estado civil; la manera como están estructurado los hogares en la actualidad, para de esa manera poder atender a ayudar a las formas de convivencia; el número de personas que trabajan, clasificarlas por actividades que realizan, en respectivos casos situación profesional, para de esa manera poder determinar en qué cantidad de personas están desempleadas y en situación de búsqueda de trabajo; de la misma manera poder determinar la cantidad de personas que pueden disponer de una educación o tienen algún nivel de estudio. Toda la información que se ha logrado recaudar se la puede utilizar para de esa manera poder planificar políticas demográficas, sanitarias, educativas, asistenciales, medioambientales y muchas otras más que llegan a ser indispensables para la población, y de la misma manera poder evaluar los resultados de las mismas. De tal forma el censo es importante, ya que, resulta ser una herramienta eficaz para la asignación de recursos económicos procedentes de los distintos órdenes del gobierno para que de esa manera se genere el desarrollo urbano y rural, incentivando así a proyectos benéficos como; la construcción de carreteras y los programas políticos social, entre otros proyectos que sean de gran beneficio para la población en general.

\section{Discusión}

Luego de hacer hincapié a la importancia que tiene los datos que genera el censo para la toma de decisiones, se puede mencionar algunos objetivos específicos tales como: 
- Proporcionar datos verídicos para de esa manera poder ubicar e identificar a los diferentes grupos de la población, tales como, regiones sociodemográficas existentes que están conformados dentro del territorio nacional.

- Es aquella que determina la información para que de esa manera las entidades públicas dependientes que son las responsables de ofrecer servicio a la sociedad obtengan datos actualizados y que sean verídicos en un periodo corto, para de la misma manera orientar y controlar de manera eficiente sus planes y programas.

- Admite de la misma manera una información actualizada de las proyecciones de la población en general a los distintos niveles territoriales.

- Se encarga de proyectar datos verídicos para de esa manera proceder al cálculo de indicadores de bienestar, de tal manera que se comprende el grado de factibilidad que nuestro país cumple. (Barreto-Villanueva, 2012)

El conteo es muy importante debido a que esta permite identificar los cambios que llega a experimentar nuestro país frente a los diferentes aspectos: demográficos, económicos y sociales, de tal manera que representa un soporte estadístico fundamental para apoyar la toma de decisiones de los diferentes sectores existentes como son público, privado y social.

Por ende, al generar un buen uso de la estadística, fomenta que la investigación llegue a ser eficiente, por lo que algunos autores como Ostle recomiendan que todos investigadores sin excepción alguna se familiaricen con ella. El papel de la estadística en la investigación representa una poderosa herramienta para las diferentes investigaciones, facilitando de tal manera el análisis correcto de los datos, y en la obtención de conclusiones a partir de ellos (Ostle, 1986).

De la misma manera se llega a comprender que la estadística es aquella que analiza o procesa una serie de datos numéricos, permitiendo a la vez el estudio de las funciones decisorias estadística y para su interpretación se basa en los índices (Badii M. A., 2004).

La estadística es aquella que genera apoyo con gran impacto a la investigación al tratar con diferentes temas que son de gran relevancia:

1. La compilación y recolección de datos.

2. El diseño de experimentos.

3. La correcta valoración, tanto de datos experimentales como detección de causa y reconocimiento.

4. El control de la calidad de la producción.

5. La identificación de diferentes parámetros de la población y suministro de diferentes medidas de la exactitud y precisión de esas ponderaciones.

6. La estimación de cualidades humanas. 
7. El análisis de conjeturas con respecto a la población general.

8. La interpretación de la relación existentes entre dos o más variables. (Badii M. J., 2007).

\section{Conclusiones}

Se concluye que la estadística es una herramienta de suma importancia en una investigación de cualquier ámbito, tales como; educativo, económico, demográfica y administración pública debido a que es la ciencia que trata de entender, organizar y tomar las mejores decisiones de acuerdo con los análisis interpretados en una investigación.

Se logró concluir también que, la estadística es aquella que tiene gran relevancia para un correcto desarrollo de un sector o una población, haciendo énfasis en el ámbito demográfico debido a que se puede analizar una información de datos verídicos gracias al censo que se lo realiza de manera habitual cada 10 años y mediante cálculos, a la vez se llega a identificar qué aspectos se puede ir cambiando o a la vez mejorando y promoviendo de esa manera una mejora de vida para la población.

Es importante reconocer que la estadística juega un papel muy importante en el desarrollo de las investigaciones, debido a que, una adecuada selección de las técnicas de análisis, garantiza confiabilidad y veracidad a la investigación realizada, por ende, es importante que el investigador tenga claro: identificar el método más apropiado para el respectivo análisis de los datos recogidos, para de esa manera poder concluir la información de una manera clara y concisa.

\section{Referencias}

<Arnal, J. D. (1992). Investigación educativa: fundamentos y metodologías. Barcelona: Editorial Labor, S.A.

Badii, M. A. (2004). Fundamentos del método. Monterrey: UANL.

Badii, M. J. (2007). Completely randomized. En M. B. Castillo, Técnicas Cuantitativas en (págs. 307-334). Monterrey: UANL.

Badii, M. J. (2007). Experimental designs. En M. B. (eds.)., Técnicas Cuantitativas en la Investigación (págs. 335-348.). Monterrey.: UANL.

Barreto-Villanueva, A. (2012). El progreso de la Estadística y su utilidad en la evaluación del desarrollo. Scielo, 15 . 
Carlos Manterola, \&. T. (2013). Por qué Investigar y Cómo Conducir una Investigación. Madrid: Int. J. Morphol.

Delgado, M. d. (2013). Actitudes y motivaciones de la capacidad emprendedora de las mujeres en España. España, Madrid: by-nc-sa.

Flores, J. G. (2003). La estadística en la investigación educativa. Revista de investigación educativa, 231-248.

Foroughbakhch, R., \& M, B. (2005). Métodos Analíticos Estadísticos. Monterrey: UANL.

Gómez Benito, J. (1987). Meta-análisis. Barcelona: PPU.

Lecompte, M. (1995). Un matrimonio conveniente: diseño de investigación cualitativa y estándares para la evaluación de programa. Revista Electrónica de Investigación y Evaluación Educativa.

Manterola, C., \& H., T. O. (2013). Por qué Investigar y Cómo Conducir una Investigación. Madrid: Int. J. Morphol.

Mendiburu. (8 de marzo de 2018). SCRIB. Obtenido de SCRIB: https://tarwi.lamolina.edu.pe/ fmendiburu/indexfiler/academic/Estadistica/parte1.pdf

Merton, R. (1964). Teoría y estructuras Sociales. México: FCE.

Ostle, B. (1986). Estadística aplicada. En B. Ostle. México: Limusa-Wiley S.A.

Parias, L. D. (mayo de 2011). saludpublicavirtual. Obtenido de saludpublicavirtual: http://saludpublicavirtual.udea.edu.co/eva/pluginfile.php/3077/mod_resource/conte nt/o/Estad\%C3\%ADstica\%2ocap\%C3\%ADtulo\%201.pdf

Ponteiro, R. (septiembre de 2013). COMPRENSION Y USO DE LA ESTADÍSTICA. Obtenido de COMPRENSION Y USO DE LA ESTADÍSTICA: https://web.cortland.edu/flteach/stats/stat-sp.html

Sánchez, J. y. (1989). Metaanálisis: una alternativa metodológica a las revisiones. En J. y. Arnau, Tratado de (págs. pp. 617-669.). Madrid: Alhambra.

Taylor, S., \& Bogdan, R. (1986). Introducción a los métodos cualitativos de investigación. Buenos Aires: Paidós. 
Zapata, H. (20 de 1 de 2017). Esup.edu.pe. Obtenido de https://www.esup.edu.pe/descargas/valotario_coem/2017/1\%20HernandezZapata\%20y\%2oMendoza-Metodologia\%2oInvestigacion.pdf

$>$

ca. 